\title{
Factor B Inhibitor LNP023
}

National Cancer Institute

\section{Source}

National Cancer Institute. Factor B Inhibitor LNP023. NCI Thesaurus. Code C156691.

An orally available, small-molecule inhibitor of complement factor B (FB) with potential immunomodulatory activity. Upon administration, FB inhibitor LNP023 binds to FB and prevents the formation of the alternative pathway (AP) C3-convertase (C3bBb). This limits the cleavage of C3 to the active frag ment C3b and may prevent C3b-mediated extravascular hemolysis in certain complement-driven disorders such as paroxysmal nocturnal hemoglobinuria (PNH). 